\title{
INTERPLANETARY CONSEQUENCES OF TRANSIENT CORONAL EVENTS OBSERVED WITH YOHKOH SXT: A CASE STUDY OF POLAR CORONAL TRANSIENT ON 15 MAY 1992
}

\author{
T. WATANABE and Y. NAKAGAWA \\ Department of Environmental Sciencies, Ibaraki University, \\ 310 Mito, Japan \\ and \\ M. KOJIMA \\ Solar-terrestrial Environment Laboratory, Nagoya University, \\ Toyokawa 442, Japan
}

November 28, 1996

\begin{abstract}
A large area $\left(5 \times 10^{10} \mathrm{~km}^{2}\right)$ of a coronal hole disappeared in concert with a transient brightening of a nearby high-latitude coronal arcade in the northern hemisphere on 15 May 1992. This coronal-hole disappearance took place in a time scale of half a day. It is suggested that the large-scale and quick change in coronal-hole geometry induced the eruption of originally closed coronal magnetic structure of the high-latitude arcade. An associated solar wind disturbance with the plasma speed of $>700 \mathrm{~km} / \mathrm{sec}$ was observed by IPS, and geomagnetic sudden commencement was reported on 18 May 1992.
\end{abstract}

\section{Introduction}

Since the launch of $\mathrm{YOHKOH}$ in late August 1991, a variety of coronal transients were observed by the Soft X-ray Telescope (SXT). Among them, large-scale arcade-like structures of soft X-ray corona which appeared often in polar region of the Sun are of interest because several authors proposed that geo-effective interplanetary disturbances were associated with coronal transients of this kind (Gosling et al., 1994; Alexander et al., 1994; McAllister et al., 1994; Watanabe et al., 1994). Although these arcade were preceded by disappearances of polar crown filaments in some cases, the reason why such a large-scale, sometimes covering the extent in the longitude of $>100^{\circ}$, was formed in a short time. In this paper, we perform a preliminary case study of a well-observed example of a polar coronal transient which took place near the north pole of the Sun on 15 May 1994 because of good data coverage. We used $\mathrm{H} \alpha$ and $\mathrm{He} 1083.0 \mathrm{~nm}$ observations for solar data and also YOHKOH SXT images. The principal data source of solar wind is the three-station IPS (interplanetary scintillation) measurements in Japan at the frequency of $327 \mathrm{MHz}$. 


\section{Solar Observations}

A transient brightening of the high-latitude corona was observed by $\mathrm{YOHKOH}$ SXT in the northern hemisphere of the Sun on May 15, 1992. Progress of the coronal transient event is seen in Fig. 1 which shows the three representative stages of evolution; the early, maximum, and late stages. The transient brightening of the corona started at about 03 UT on 15 May, and reached to its maximum level at about $11 \mathrm{UT}$ on the same date. The enhancement of coronal brightness was still recognized even 14 hours after the initiation of the brightening (bottom). It seems that the brightest portion of the coronal transient apparently located on the top of a large-scale arcade structure overlying the polar-crown neutral line running along the latitudes of $40^{\circ} \mathrm{N}$ $60^{\circ} \mathrm{N}$ (Solar-Geophys. Data, 1992a). According to preliminary survey of $\mathrm{H} \alpha$ monitors in Japan, no remarkable $\mathrm{H} \alpha$ event was observed around the arcade in question. We checked also Kitt Peak He $1083.0 \mathrm{~nm}$ images taken on 14-16 May 1992, but no appreciable change in dark filament geometry was found in this region.

The most outstanding coronal event apparently relating to this transient coronal brightening is the disappearance of a large portion of a mid-latitude coronal hole seen in the daily maps of He $1083.0 \mathrm{~nm}$ line, as shown in Fig. 2 (Solar-Geophys. Data, 1992b). It should be noted that the He image on 15 May 1992 was taken at 15:21 UT in the maximum phase of the coronal brightening. Comparing these maps, it is noticed that a large part of the northern end of the elongated coronal hole which was situated near the central meridian of the solar disk on 15 May disappeared by the observational time on 16 May.

Since the close connection between the transient brightening of the arcade and the disappearing coronal hole is suggested, we trace the change in the area of the disappearing coronal hole and the development of the coronal brightening, using YOHKOH SXT images. To do this, we selected a portion of the coronal hole to follow the change in the characteristic area on "flattened" SXT A1.1 images, as shown in Fig. 3. These images are reproduced from original SXT images by converting these spherical images to flat images. To trace the time variation of the soft X-ray brightness of the coronal brightening, we integrated the outputs of $5 \times 5$ pixels centred at a fixed point where the brightness of the arcade showed the maximum level. The location of the center is also shown in Fig. 3. We see only the relative change in the X-ray brightness taking the observed maximum brightness is $100 \%$. The results are shown in Fig. 4. It is seen in this figure that the brightening of the arcade was observed approximately in concert with the decrease in the coronal-hole area and that the brightness of the arcade faded out when the decrease in the area stopped at about 12 hours after the initiation. The rate of aerial change of the coronal hole is about $4 \times 10^{9} \mathrm{~km}^{2} / \mathrm{hr}$. 


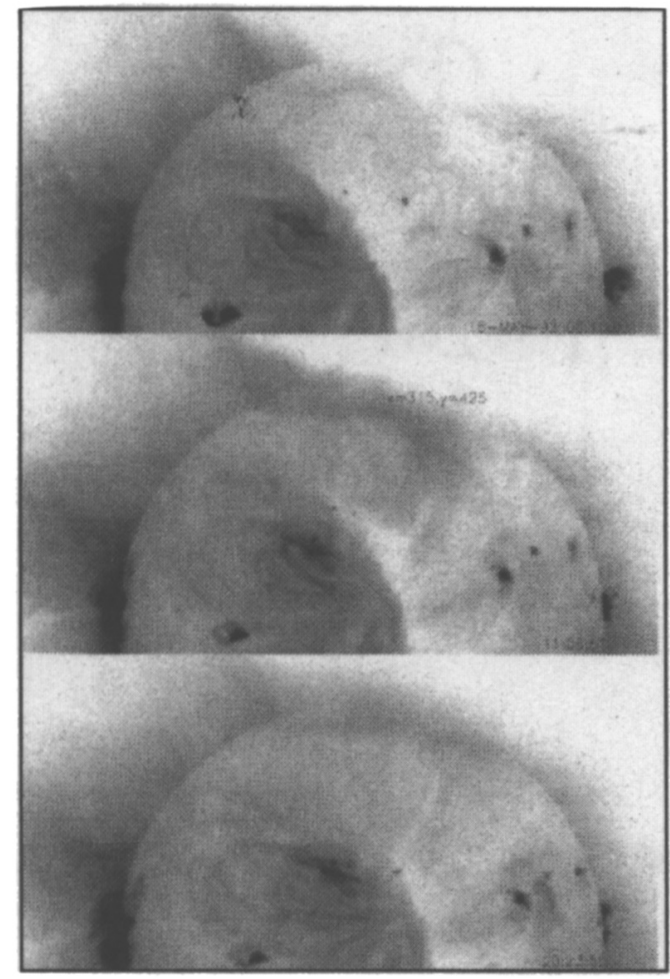

Fig. 1. Selected YOHKOH SXT images (A1.1) of the northern half of the Sun showing the evolution of large-scale brightening of the high-latitude coronal arcade on 15 May 1992; 06:10:03 UT (top), 11:06:51 UT (middle), and 20:23:55 UT (bottom).
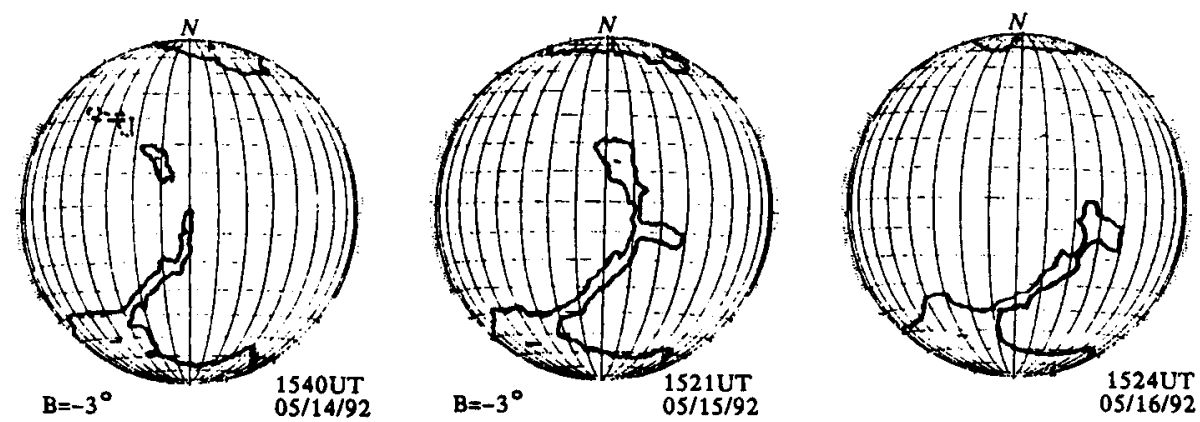

Fig. 2. Kitt-Peak daily maps of He 1083.0nm coronal holes taken on 14 - 16 May 1992 (Solar-Geophys. Data, 1992). 


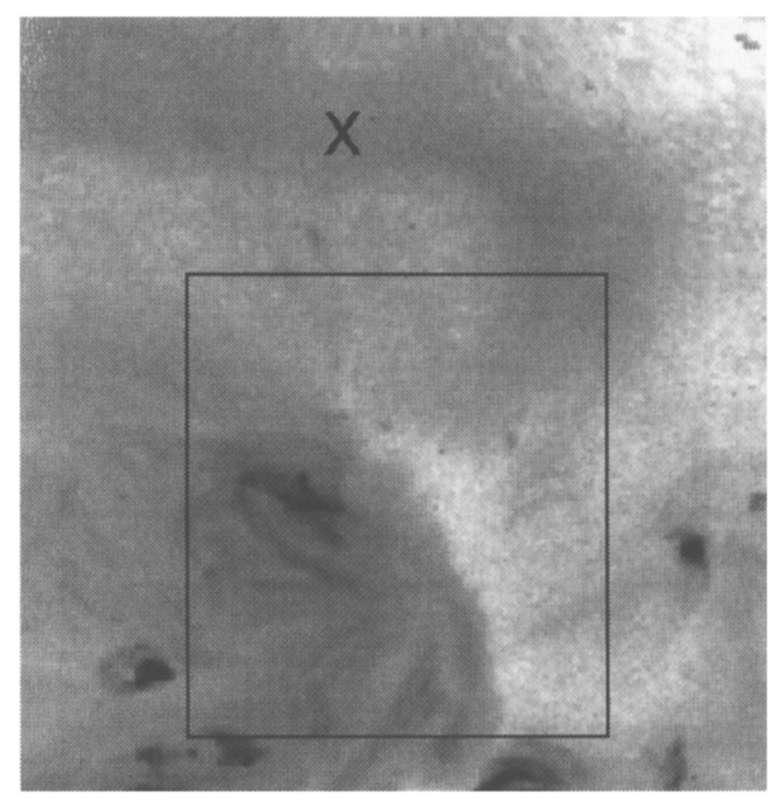

Fig. 3. The domain in which the time variation of the characteristic area of the coronal hole is measured is indicated by the box. The center of the $5 \times 5$ pixels selected to estimate the relative time-variation of the soft X-ray brightness to follow the evolution of the transient brightening of the high-latitude arcade is shown by the cross.

We conclude from observations mentioned above that the disappearing coronal-hole was the principal agent to produce the transient brightening of the arcade, through the magnetic reconnection process. It is also inferred from soft X-ray observations that the transient brightening of the arcade indicated the presence of an outgoing CME, as seen in many cases of filament disappearances (e.g. Watanabe et al., 1994; Kozuka et al., 1995). The presence of the CME can be confirmed using interplanetary observations as discussed in the next section.

\section{Interplanetary Observations}

A transient increase in the solar wind speed up to $707 \mathrm{~km} / \mathrm{sec}$ was observed by IPS of $0221+07$ at 0.31 AU from the Sun at 17 UT on 16 May 1992 (Fig. 4). A geomagnetic sudden commencement of a storm (sc) was observed at 20:21 UT on 18 May. When we assume that the sc was caused by the interplanetary disturbance relating to the transient coronal event on 15 May, the average speed of the disturbance between the Sun and the Earth is estimated to be about $530 \mathrm{~km} / \mathrm{sec}$. This speed is typical to interplanetary disturbances associated with filament-disappearance events. 


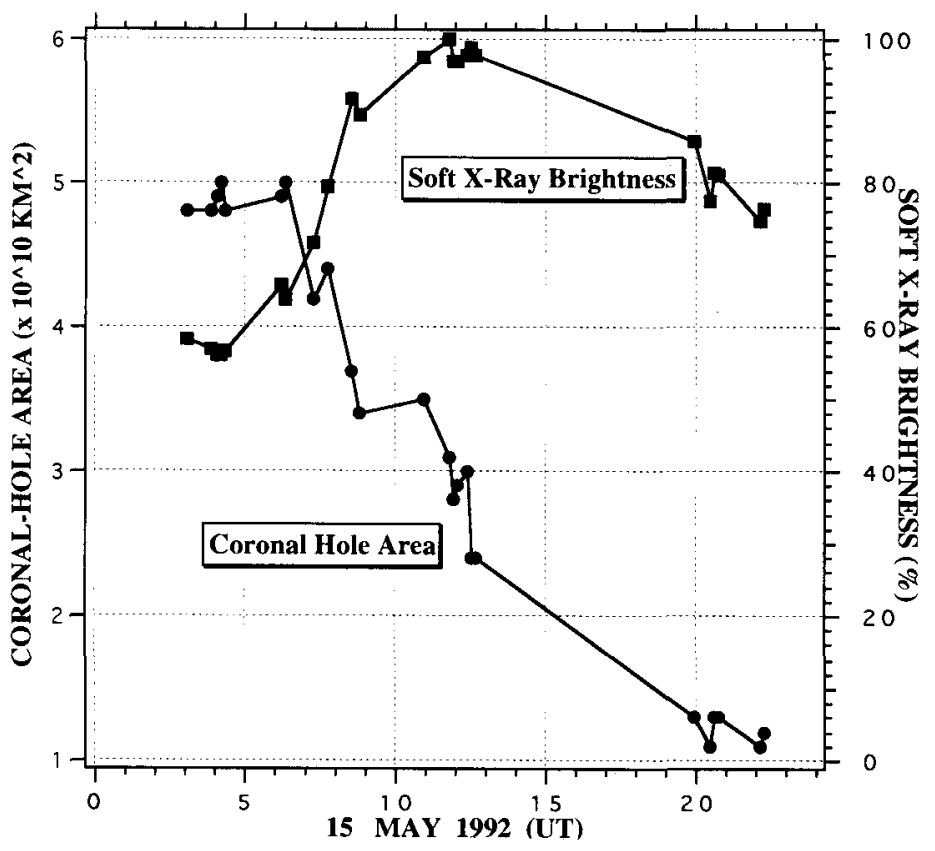

Fig. 4. Time variation of the characteristic area of the coronal hole in the region shown in Fig. 3 (filled circles) and that of the integrated brightness of the $5 \times 5$ pixels shown also in Fig. 3 (filled squares). The maximum brightness is set to be $100 \%$.

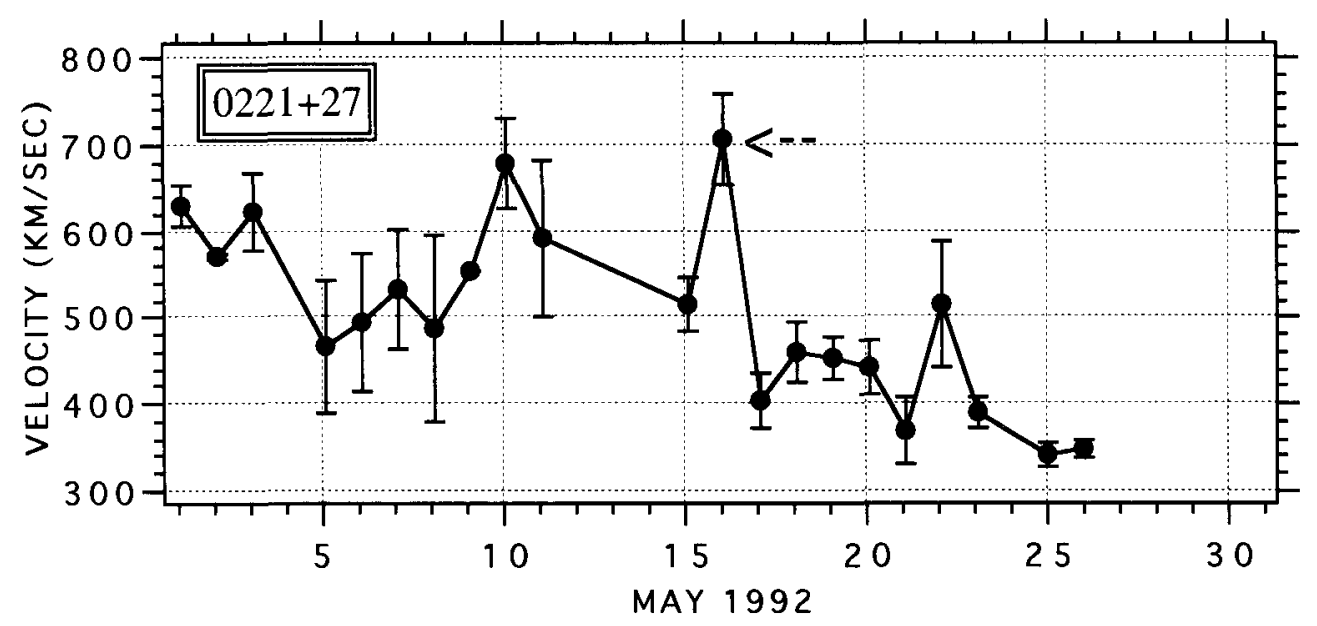

Fig. 5. Solar wind speed measurements by IPS of $0221+27$ in May 1992. An enhancement of the solar wind speed observed immediately after the transient brightening of the high-latitude arcade on 15 May 1992 is indicated by the arrow. 


\section{Concluding Remarks}

It has been seen that the transient brightening of a high-latitude coronal arcade on 15 May 1992 took place when the northern portion of the elongated coronal hole, originally located immediately to the south of the place of the brightening, disappeared in a short time (about 12 hours). The area of disappeared portion is about $5 \times 10^{10} \mathrm{~km}^{2}$ (about $2 \%$ of the total hemispherical area of the Sun), and the rate of aerial changes is about $4 \times 10^{9} \mathrm{~km}^{2} / \mathrm{hr}$. Such a quick and large-scale change in the coronal-hole geometry will not be produced by a dynamical effect of an outgoing material being proposed for some disappearing-filament events; a local change in the dynamo process to produce the coronal hole field will be conceived. Since the maximum brightness of the arcade took place in the course of the disappearance of the coronal hole, it is suggested that the magnetic reconnection process taking place above the arcade was driven by the changing magnetic geometry of the coronal hole. A geo-effective CME was also produced in the course of the disappearing coronal-hole event. Prediction of such a kind of events will be difficult only by $\mathrm{H} \alpha$ monitor.

It is not clear in this stage how often such a disappearing coronal-hole was observed for transient brightening of high-latitude arcades. Further detailed study is in progress.

\section{Acknowledgements}

The soft X-ray images are from YOHKOH mission of Institute of Space and Astronautical Sciences, Japan. The authors acknowledge YOHKOH team members who are conducting the daily tracking of the spacecraft.

\section{References}

Alexander, D., Greg, L., Slater, Hudson, H.S., McAllister, A.H., and Harvey, K.L.:1994, 'The Large Scale Coronal Eruptive Event of April 14 1994', 3rd SOHO Workshop, Solar Dynamic Phenomena and Solar Wind Consequences, Proceedings, SP-373, 187-190

Gosling, J.T., McComas, D.J., Phillips, J.L., Weiss, L.A., Pizzo, V.J. Goldstein, B.E., and Forsyth, R.J.: 1994, 'A New Class of Forward-Reverse Shock Pairs in the Solar Wind', Geophys. Res. Lett., 2271-2274

Kozuka, Y., Watanabe, T., Kojima, M., Ohyama, M., Tsuneta, S., Khan, J.I., and Watari, S.: 1994, 'The Dynamical Characteristics of a Disappearing-Filament Associated Interplanetary Disturbance Observed in 1992 early May', Publ. Astron. Soc. Japan, 47, 377-381

McAllister, A., Singer, H., Dryer, M., McIntosh, P., and Wiess, L.: 1994, 'A Large Polar Crown CME and a Severe Geomagnetic Storm: April 14-17, 1994', 3rd SOHO Workshop, Solar Dynamic Phenomena and Solar Wind Consequences, Proceedings, SP-373, 315-318 
Solar-Geophysical Data, SESC PRF 873, U.S. Department of Commerce, Boulder, CO 80303 (1992a)

Solar-Geophysical Data, SESC PRF 872, U.S. Department of Commerce, Boulder, CO 80303 (1992b).

Watanabe, T., Kojima, M., Kozuka, Y., and Yatagai, H.: 1994, 3rd SOHO Workshop, Solar Dynamic Phenomena and Solar Wind Consequences, SP-373, 269-274

Watanabe, T., Kozuka, Y., Ohyama, M., Kojima, M., Yamaguchi, K., Watari, S., Tsuneta, S., Joselyn, J.A., Harvey, K.L., Acton, L., and Klimchuk, J.A.: 1992, Publ. Astron. Soc. Japan, 44, L199-L202 